FERMILAB CONF-04-302-T; IPPP 04/69; PITHA 04/16

October 2004

\title{
Towards pair production near threshold with unstable particle effective theory*
}

\author{
M. Beneke ${ }^{\mathrm{a}}$, N. Kauer $^{\mathrm{a}}$, A. Signer ${ }^{\mathrm{b}}$, G. Zanderighi $^{\mathrm{c}}$ \\ ${ }^{a}$ Institut für Theoretische Physik E, RWTH Aachen, D-52056 Aachen, Germany \\ ${ }^{\mathrm{b}} \mathrm{IPPP}$, Department of Physics, University of Durham, Durham DH1 3LE, England \\ ${ }^{\mathrm{c}}$ Fermi National Accelerator Laboratory, Batavia, IL 60510-500, USA
}

We illustrate the use of effective theory techniques to describe processes involving unstable particles close to resonance. First, we present the main ideas in the context of a scalar resonance in an Abelian gauge-Yukawa model. We then outline the necessary modifications to describe $W$-pair production close to threshold in electronpositron collisions.

\section{Introduction}

Processes involving heavy, unstable particles, play an important role in precision tests of the Standard Model and its hypothetical extensions. Typically these particles are studied in processes where they are produced close to resonance. It is thus important to overcome the difficulties related to the breakdown of ordinary weak-coupling perturbation theory in the description of such processes.

As is well known, the singularity of the intermediate propagator can be avoided if the width, $\Gamma$, of the unstable particle is taken into account through self-energy resummation. However, this alone does not guarantee an accurate description of the process. This is partly reflected by the fact that such a procedure may produce gaugedependent results. Most approaches that have been put forward (for a summary see [1]) so far are focused on this particular aspect of the problem. Recently, we proposed a different approach 2 making use of effective theory methods. This allows to perform a systematic expansion in the coupling as well as in $\Gamma / M$, where $M$ is the mass of the unstable particle. The main advantage of this method is that it provides us with a

\footnotetext{
*Invited talk given by A.S. at the 11 th International Conference on Quantum Chromodynamics, Montpellier, France (5-10th July 2004)
}

computational scheme for improving the accuracy of calculations in a systematic way, where gauge invariance is automatic.

\section{Effective Theory}

At the heart of this method lies the observation that there is a hierarchy of scales between the mass and the width of the unstable particle. We exploit this hierarchy by constructing an effective theory and integrating out the modes that are not needed for the description of the external state. A first step to apply these methods in the context of unstable particles has been made in [3] and, as discussed in the next section, the programme has been carried out explicitly for a toy model in [2].

In technical terms, we identify the relevant modes and use the method of regions [4] to expand the integrals in $\Gamma / M$. Together with the standard expansion in the coupling $\alpha$ we thus achieve a systematic organization of the calculation in a series in $\alpha$ and $\Gamma / M$.

As a first step in this procedure we integrate out hard momenta $k \sim M$. The effects of the hard momenta are incorporated into the matching coefficients, leaving the effective theory without dynamical hard modes. This is reminiscent and in fact often parallel to similar developments in other effective theories such as non-relativistic QCD (NRQCD), heavy quark 
effective theory (HQET) or soft-collinear effective theory (SCET). The hard corrections are so called factorizable corrections, whereas the nonfactorizable corrections are reproduced in the effective theory by the still dynamical modes 3 . The precise nature of these modes depends to some extent on the underlying theory and even on the observable in question. In the next sections we will give a more detailed discussion, first for a toy model, then for $W$-pair production near threshold.

\section{An Abelian gauge-Yukawa model}

In order to outline the main steps in the construction of the effective theory (full details can be found in 2] ) we consider a toy model, consisting of a heavy, scalar field $\phi$ that decays through a Yukawa interaction into an "electron" and "neutrino". The electron field, $\psi$, as well as the scalar field are charged under an Abelian gauge group. The Lagrangian is given by

$$
\begin{aligned}
\mathcal{L}= & \left(D_{\mu} \phi\right)^{\dagger} D^{\mu} \phi-\hat{M}^{2} \phi^{\dagger} \phi+\bar{\psi} i \not D \psi+\bar{\chi} i \not \partial \chi \\
& -\frac{1}{4} F^{\mu \nu} F_{\mu \nu}-\frac{1}{2 \xi}\left(\partial_{\mu} A^{\mu}\right)^{2} \\
& +y \phi \bar{\psi} \chi+y^{*} \phi^{\dagger} \bar{\chi} \psi-\frac{\lambda}{4}\left(\phi^{\dagger} \phi\right)^{2}+\mathcal{L}_{\mathrm{ct}},
\end{aligned}
$$

where $\hat{M}$ and $\mathcal{L}_{\text {ct }}$ denote the renormalized mass and the counterterm Lagrangian respectively.

For the purpose of illustration we outline the calculation of the totally inclusive cross section of the process $\bar{\nu}(q) e^{-}(p) \rightarrow X$ as a function of $s \equiv(p+q)^{2}$ with $s-M^{2} \sim M \Gamma \sim \alpha M^{2}$, where $\alpha$ denotes collectively the gauge and Yukawa couplings. We obtain the cross section as the imaginary part of the forward scattering amplitude $\mathcal{T}(s)$. The aim is to compute $\mathcal{T}$ through a systematic expansion in $\alpha$ and

$\delta \equiv \frac{s-\hat{M}^{2}}{\hat{M}^{2}} \sim \frac{\Gamma}{\hat{M}} \sim \alpha$.

As a first step, we integrate out the hard modes with $k \sim M$. In the resulting effective theory, the effects of the hard modes are included in the matching coefficients of the operators. For the heavy scalar, this procedure is equivalent to the construction of HQET. In fact we write the momentum of the scalar particle near resonance as $P^{\mu}=\hat{M} v^{\mu}+k^{\mu}$ where $v^{2}=1$ and $k \sim M \delta$ and define the soft field $\phi_{v}$ (called "resonant" in [3]) by removing the rapid spatial variation $e^{-i \hat{M} v \cdot x}$ from the $\phi$-field. Thus, a soft field has momentum $k \sim M \delta$. We also define the matching coefficient

$\Delta \equiv \frac{\bar{s}-\hat{M}^{2}}{\hat{M}}$

where $\bar{s}$ is the complex pole of the propagator. Since $\bar{s}$ and $\hat{M}$ are gauge invariant, $\Delta$ is guaranteed to be gauge invariant as well. As required for a matching coefficient, $\Delta$ is given entirely by hard contributions. In fact, expanding the hard part of the self energy, $\Pi_{h}(s)$, as

$\Pi_{h}(s)=\hat{M}^{2} \sum_{k, l} \delta^{l} \Pi^{(k, l)}$

with $\Pi^{(k, l)} \sim \alpha^{k}$, we can write the matching coefficient as

$$
\frac{\Delta}{\hat{M}}=\Pi^{(1,0)}+\left(\Pi^{(2,0)}+\Pi^{(1,1)} \Pi^{(1,0)}\right)+\ldots
$$

The explicit form of the matching coefficient depends on the renormalization scheme. In the pole scheme $\Delta=-i \Gamma$.

The terms of the effective Lagrangian bilinear in $\phi_{v}$ can be written as

$$
\begin{aligned}
\mathcal{L}_{\phi \phi} & =2 \hat{M} \phi_{v}^{\dagger}\left(i v \cdot D_{s}-\frac{\Delta}{2}\right) \phi_{v} \\
& +2 \hat{M} \phi_{v}^{\dagger}\left(\frac{\left(i D_{s} \top\right)^{2}}{2 \hat{M}}+\frac{\Delta^{2}}{8 \hat{M}}\right) \phi_{v}+\ldots,
\end{aligned}
$$

where we defined $D_{s \top}^{\mu} \equiv D_{s}^{\mu}-\left(v \cdot D_{s}\right) v^{\mu}$. The interactions of the $\phi_{v}$-field with soft photons can be incorporated through the soft covariant derivative $D_{s} \equiv \partial-i g A_{s}$, because the separation into hard and soft parts respects gauge invariance.

The Lagrangian Eq. (6) describes the propagation of an unstable scalar particle close to resonance. The first line is the leading term and the inclusion of $\Delta / 2$ into the propagator corresponds to self-energy resummation. The terms on the second line are suppressed by one power of $\delta$ and contribute at NLO. Terms that are suppressed by further powers of $\delta$ can be included systematically, if required. 
In order to complete the construction of the effective Lagrangian we have to include kinetic terms for the soft and collinear photons and fermions, as well as the production and decay vertices for the unstable particle. In the present Yukawa model the NLO line shape is completely described by the addition of the production and decay vertices

$$
\begin{aligned}
\mathcal{L}_{\text {int }} & =C\left[y \phi_{v} \psi_{n_{-}} \chi_{n_{+}}+\text {h.c. }\right] \\
& +\frac{y y^{*} D}{4 \hat{M}^{2}}\left(\bar{\psi}_{n_{-}} \chi_{n_{+}}\right)\left(\bar{\chi}_{n_{+}} \psi_{n_{-}}\right)
\end{aligned}
$$

where the matching coefficient $C$ must be computed to one loop, and $D=1$ at tree level. In this form of writing the effective Lagrangian we have integrated out the collinear modes, leaving only soft and external-collinear modes in the Lagrangian. The latter describe soft fluctuations around the external light-like momenta. Finally, one must compute the scattering process to the one-loop order in the effective theory. We refer to 2 for a thorough discussion.

Outside the kinematic region $\delta \sim \alpha$ the effective theory breaks down. To obtain a consistent description for all values of $s$, the result of the effective theory has to be matched to an offresonance calculation in the full theory.

\section{4. $W$-pair production close to threshold}

The toy model presented above is in many ways much simpler than the Standard Model, but it contains all the relevant features of resonant particle production. Applying the same techniques to a realistic process may result in additional technical complications, but no new conceptional difficulties arise.

An important application is $W$-pair production close to threshold at an electron-positron collider. This process is crucial for the precise determination of the $W$ mass and has been thoroughly studied away from threshold. In particular, the oneloop electroweak corrections have been computed in the double pole approximation (DPA) [5]. Here we focus on obtaining results that are valid near threshold, where the DPA is supposed to break down.

As mentioned before, the first step is to inte- grate out the hard modes. For pair production close to threshold, the $W$ bosons are then described by a non-relativistic Lagrangian, analogous to NRQCD and the complications due to the Coulomb singularity can be addressed using standard methods. We are thus left with dynamical degrees of freedom [4] familiar from NRQCD [6]. We particularly emphasize the presence of soft and potential gauge bosons. Working in the center of mass frame, the typical momentum of a $W$ with velocity $v$ is $|\vec{k}| \sim M_{W} v$ and the typical (non-relativistic) energy is $E \sim M_{W} v^{2}$. This corresponds to a potential mode. For soft modes, the energy and momentum scale as $M_{W} v$. The initial state fermions and the decay products are described by collinear modes familiar from SCET [7]. In fact we will need several collinear modes, one for each direction present in the process under consideration.

To be specific, let us consider the process

$e^{-}\left(p_{1}\right) e^{+}\left(p_{2}\right) \rightarrow \mu^{-}\left(l_{1}\right) \bar{\nu}_{\mu}\left(l_{2}\right) u\left(l_{3}\right) \bar{d}\left(l_{4}\right)$

with $\left(l_{1}+l_{2}\right)^{2}-M_{W}^{2} \sim\left(l_{3}+l_{4}\right)^{2}-M_{W}^{2} \sim M_{W}^{2} v^{2} \sim$ $M_{W} \Gamma_{W}$. By assumption $\sqrt{s} / 2-M_{W} \sim M_{W} \Gamma_{W}$.

At leading order, the description of the process can be split into three parts. First, the $W$-pair is produced, then it propagates, and finally the $W$ bosons decay. At higher orders, this simple picture is complicated by the exchange of Coulomb photons, of photons that connect the various stages and/or by the presence of additional photons in the final state. Furthermore, singleresonant and non-resonant diagrams have to be taken into account. We count $\alpha_{s} \sim v \sim \sqrt{\alpha_{e w}}$ and by next-to-leading order (NLO) contributions we understand the $\mathcal{O}\left(\alpha_{\text {ew }}\right) \sim \mathcal{O}\left(v^{2}\right) \sim \mathcal{O}\left(\alpha_{s}^{2}\right)$ corrections. In this article we present all contributions that are needed at $\sqrt{\mathrm{NLO}}$, that is, all corrections of order $v \sim \alpha_{s}$.

Starting with the construction of the nonrelativistic Lagrangian we first remark that the gauge dependence of the vector-boson propagator is not an issue. Indeed, expanding the momentum of a non-relativistic $W$ as $k^{\mu}=M_{W} v^{\mu}+q^{\mu}$, where $v^{\mu} \equiv(1, \overrightarrow{0})$, and $q^{\mu}=\left(q^{0}, \vec{q}\right)$ and taking into account $q^{0} \sim M_{W} v^{2}$ and $|\vec{q}| \sim M_{W} v$ the propagator in a general $R_{\xi}$ gauge upon expansion 
becomes

$$
\begin{array}{r}
\frac{-i}{k^{2}-M_{W}^{2}}\left(g^{\mu \nu}-(1-\xi) \frac{k^{\mu} k^{\nu}}{k^{2}-\xi M_{W}^{2}}\right) \\
\rightarrow \quad \frac{-i\left(g^{\mu \nu}-v^{\mu} v^{\nu}\right)}{k^{2}-M_{W}^{2}} \rightarrow \frac{i \delta^{i j}}{2 M_{W} q^{0}-\vec{q}^{2}} .
\end{array}
$$

The propagator scales as $v^{-2}$, is gauge independent and describes three polarization states of a non-relativistic particle. This is consistent with the fact that the degrees of freedom with mass $\sqrt{\xi} M_{W}$ have been integrated out. (In Feynman gauge the propagator is given by $-i g^{\mu \nu} /\left(2 M_{W} q^{0}-\vec{q}^{2}\right)$. The effects of the unphysical scalar polarization are cancelled by the pseudo-Goldstone field, which has mass $M_{W}$ and is still present in the effective theory.) Including the decay width we obtain for the non-relativistic Lagrangian up to NLO

$$
\begin{aligned}
\mathcal{L}_{n r} & =\sum_{\mp} \Omega_{\mp}^{* i}\left(i D^{0}+\frac{\vec{D}^{2}}{2 \hat{M}_{W}}-\frac{\Delta}{2}\right) \Omega_{\mp}^{i} \\
& +\sum_{\mp} \Omega_{\mp}^{* i} \frac{\left(\vec{D}^{2}-\hat{M}_{W} \Delta\right)^{2}}{8 \hat{M}_{W}^{3}} \Omega_{\mp}^{i},
\end{aligned}
$$

where $\Omega_{ \pm}$denote the non-relativistic vector fields with mass dimension $3 / 2$ for the $W^{ \pm}$bosons. The matching coefficient $\Delta \equiv\left(\bar{s}-M_{W}^{2}\right) / M_{W}$ is defined as before. Its leading part, $\Delta^{(1)} \sim M_{W} \alpha_{e w}$ scales as $D^{0}$ and $\vec{D}^{2}$ and, thus, has to be included in the leading order Lagrangian. Accordingly, the propagator of a $\Omega_{ \pm}$with energy $E$ and momentum $\vec{k}$ is given by

$$
\frac{i \delta^{i j}}{\left(E-\frac{\vec{k}^{2}}{2 M_{W}}-\frac{\Delta^{(1)}}{2}\right)} \text {. }
$$

Higher order corrections to $\Delta$ can either be resummed, i.e. included in the propagator, or included perturbatively as interactions. At $\sqrt{\mathrm{N}} \mathrm{LO}$ we need $\Delta^{(3 / 2)}$, the $\mathcal{O}\left(\alpha_{e w} \alpha_{s}\right)$ corrections to $\Delta$. Higher order corrections are at least NLO as are the terms in the second line of Eq. (10).

The production of a $W^{-} W^{+}$pair is described by effective vertices to be added to $\mathcal{L}_{n r}$. In order to obtain the corresponding operators and their matching coefficients, we have to compute the amputated, renormalized on-shell Green function for $e^{+} e^{-} \rightarrow W^{+} W^{-}$to the desired order in ordinary weak-coupling perturbation theory. At leading order, only the helicity configuration $e_{L}^{-} e_{R}^{+}$ contributes and the corresponding operator reads

$\mathcal{L}_{p}^{(0)}=\frac{\pi \alpha_{e w}}{M_{W}^{2}}\left(\bar{e}_{L} \gamma^{[i} n^{j]} e_{L}\right)\left(\Omega_{-}^{* i} \Omega_{+}^{* j}\right)$,

where $e_{L}\left(\bar{e}_{L}\right)$ is an external-collinear field 2 with large momentum in the $\vec{n}(-\vec{n})$ direction and we introduced the notation $a^{[i} b^{j]} \equiv a^{i} b^{j}+a^{j} b^{i}$. Including terms that are suppressed by one power of $v$ we get additional operators

$$
\begin{aligned}
\mathcal{L}_{p}^{(1 / 2)} & =\frac{c_{1}}{M_{W}^{3}}\left(\bar{e}_{L} \gamma^{j} e_{L}\right)\left(\Omega_{-}^{* i}(-i) D^{j} \Omega_{+}^{* i}\right) \\
& +\frac{c_{2}}{M_{W}^{3}}\left(\bar{e}_{L} \gamma^{[i} e_{L}\right)\left(\Omega_{-}^{* i}(-i) D^{j]} \Omega_{+}^{* j}\right) \\
& +\frac{c_{3}}{M_{W}^{3}}\left(\bar{e}_{L} \gamma^{[i} n^{j]} n^{l} e_{L}\right)\left(\Omega_{-}^{* i}(-i) D^{l} \Omega_{+}^{* j}\right) \\
& +\frac{c_{4}}{M_{W}^{3}}\left(\bar{e}_{L} \gamma^{j} \gamma^{l} \gamma^{i} e_{L}\right)\left(\Omega_{-}^{* i}(-i) D^{l} \Omega_{+}^{* j}\right)
\end{aligned}
$$

with the matching coefficients

$$
\begin{aligned}
& c_{1}=\pi \alpha_{e w} \frac{M_{Z}^{2} \sin ^{2} \theta_{w}-2 M_{W}^{2}}{4 M_{W}^{2}-M_{Z}^{2}} \\
& c_{2}=\pi \alpha_{e w} \frac{M_{Z}^{2}\left(1-2 \sin ^{2} \theta_{w}\right)}{4 M_{W}^{2}-M_{Z}^{2}} \\
& c_{3}=2 \pi \alpha_{e w} \\
& c_{4}=\pi \alpha_{e w}
\end{aligned}
$$

All derivatives on the potential $W^{ \pm}$fields in Eq. (13) scale as $D^{i} \sim M_{W} v$. We also remark that the $e_{R}^{-} e_{L}^{+}$helicity configuration does not vanish at $\sqrt{\mathrm{N} L O}$. Consequently, there are additional operators in $\mathcal{L}_{p}^{(1 / 2)}$ involving $\bar{e}_{R}$ and $e_{R}$.

The decay of the vector bosons can be described in a similar way as the production, namely through decay vertices. The operators with their matching coefficients are obtained through matching of the corresponding on-shell Green function. For the leptonic decay, at $\sqrt{\mathrm{NLO}}$ there are no loop corrections to be taken into account, whereas for the hadronic decay, there are corrections of the order $\alpha_{s} \sim v$. When the calculation is completely inclusive on the hadronic decay products, these corrections, together with the corrections from final state emission of an additional 
gluon, are taken into account by the hadronic part of the decay width. For the decay operators that are relevant to the process Eq. (8) we obtain

$\mathcal{L}_{d}^{(0)}=-\frac{g_{e w}}{2 \sqrt{M_{W}}}\left(\Omega_{-}^{i} \bar{\mu}_{L} \gamma^{i} \nu_{L}+\Omega_{+}^{i} \bar{u}_{L} \gamma^{i} d_{L}\right)$.

Kinematic corrections to $\mathcal{L}_{d}^{(0)}$ are suppressed by at least two powers of $v$.

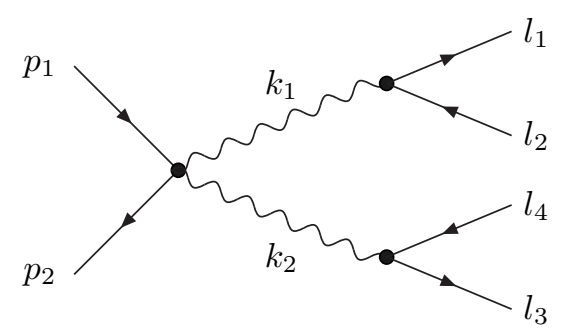

Figure 1. Leading order Feynman diagram in the effective theory.

We are now in a position to compute the leading order amplitude within the effective theory. The corresponding diagram is depicted in Figure 1 For the vertices we have to take the leading order production and decay vertices, Eqs.(12) and (18). The propagators of the intermediate vector bosons are given by Eq.(11). Putting everything together we obtain

$\mathcal{A}^{(0)}=\frac{i \alpha_{e w}^{2} \pi^{2}}{M_{W}^{3}}\left\langle p_{2}-\left|n^{[i} \gamma^{j]}\right| p_{1}-\right\rangle \times$

$\frac{\left\langle l_{1}-\left|\gamma^{i}\right| l_{2}-\right\rangle\left\langle l_{3}-\left|\gamma^{j}\right| l_{4}-\right\rangle}{\left(E_{1}-\frac{\left(\vec{l}_{1}+\vec{l}_{2}\right)^{2}}{2 M_{W}}-\frac{\Delta^{(1)}}{2}\right)\left(E_{2}-\frac{\left(\vec{l}_{3}+\vec{l}_{4}\right)^{2}}{2 M_{W}}-\frac{\Delta^{(1)}}{2}\right)}$,

where $E_{1} \equiv l_{1}^{0}+l_{2}^{0}-M_{W}, E_{2} \equiv l_{3}^{0}+l_{4}^{0}-M_{W}$ and we used standard helicity notation.

Turning to the calculation of the $\sqrt{\mathrm{N}} \mathrm{LO}$ amplitude, we also have to consider the helicity configuration $e_{R}^{-} e_{L}^{+}$. However, the corresponding calculation is analogous to the calculation of $\mathcal{A}^{(0)}$, barring the replacement of the production vertex. Let us, therefore, focus on the corrections needed in the case of $e_{L}^{-} e_{R}^{+}$.

For all stages, production, propagation and decay we have to include corrections. In the case of the production stage, we have to include diagrams as shown in Figure 1 with the production vertex due to operators given in Eq. (13) rather than Eq.(12). For the propagation stage, we need to include the $\mathcal{O}\left(\alpha_{e w} \alpha_{s}\right)$ corrections to $\Delta$. If resummed, this results in a change in the propagator $\Delta^{(1)} \rightarrow \Delta^{(1)}+\Delta^{(3 / 2)}$. Finally, regarding the decay stage, we mention again that the $\mathcal{O}\left(\alpha_{s}\right)$ corrections to the decay vertex are not explicitly needed in a completely inclusive calculation.

Apart from these trivial corrections, there is a further contribution at $\sqrt{\mathrm{N}} \mathrm{LO}$, namely the exchange of a single Coulomb (potential) photon, shown in Figure 2 This contribution is suppressed by $\alpha / v \sim v$ relative to the leading order amplitude. Thus, contrary to top pair production close to threshold, these contributions can be treated perturbatively and need not be summed. This is the only correction at $\sqrt{\mathrm{N} L O}$ that is not due to hard modes.

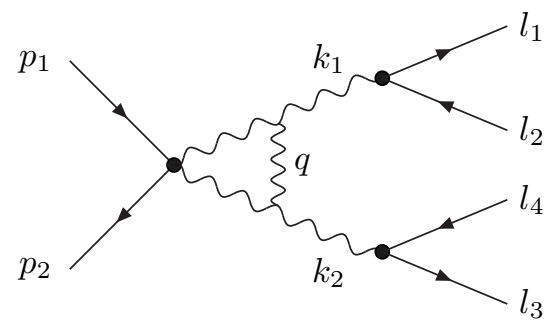

Figure 2. The exchange of a single Coulomb photon is suppressed by $\alpha / v$ relative to the leading order amplitude and, thus, contributes at $\sqrt{N} L O$.

The momentum of the potential Coulomb photon scales as $q^{0} \sim M_{W} v^{2},|\vec{q}| \sim M_{W} v$. Thus the propagator is given by $i / \vec{q}^{2}$. Reading off the Feynman rules of the $W W \gamma$ vertex of Eq. (10), we obtain for the amplitude due to single photon exchange

$$
\begin{aligned}
& \mathcal{A}^{(1 / 2, c)}=-i(4 \pi \alpha) \mathcal{A}^{(0)} \times \\
& \int \frac{d^{D} q}{(2 \pi)^{D}} \frac{1}{\vec{q}^{2}} \frac{1}{\left(E_{1}-q^{0}-\frac{\left(\vec{k}_{1}-\vec{q}\right)^{2}}{2 M_{W}}-\frac{\Delta^{(1)}}{2}+i \epsilon\right)} \\
& \frac{1}{\left(E_{2}+q^{0}-\frac{\left(\vec{k}_{2}+\vec{q}\right)^{2}}{2 M_{W}}-\frac{\Delta(1)}{2}+i \epsilon\right)},
\end{aligned}
$$

where $\vec{k} \equiv \vec{k}_{1}=\vec{l}_{1}+\vec{l}_{2}$ and $\vec{k}_{2}=\vec{l}_{3}+\vec{l}_{4}=-\vec{k}$. 
After performing the $q^{0}$ contour integral, the $\vec{q}$ integration is straightforward and we obtain

$$
\begin{aligned}
& \mathcal{A}^{(1 / 2, c)}=\mathcal{A}^{(0)} \frac{\alpha M_{W}}{|\vec{k}|} \times \\
& \arctan \frac{|\vec{k}|}{\sqrt{M_{W}\left(\Delta^{(1)}-E_{1}-E_{2}\right)-i \epsilon}},
\end{aligned}
$$

in agreement with 8 .

We note that it is possible to resum the contributions due to multiple potential photon exchange and derive a Green function for the $W$ pair. This is described most naturally within the context of a potential non-relativistic Lagrangian, analogous to potential non-relativistic QED [9].

\section{Conclusions}

The fundamental reason for the breakdown of ordinary weak-coupling perturbation theory for processes involving resonant unstable particles is the appearance of a second small scale. We described a method that overcomes these problems using an effective theory approach and applied this first to a toy model involving an unstable scalar particle and then to $W$-pair production near threshold.

The applications we presented are technically rather simple. In the case of $W$-pair production this is because we have considered only the $\mathcal{O}(v)$ corrections. However, we would like to stress once more, that including higher-order corrections introduces only technical problems and no new conceptual problems arise.

In this article, we have restricted ourselves to a totally inclusive cross section. More complicated final state kinematics requires in general the introduction of additional modes in the effective theory. Also, phase-space integrals have to be expanded. This is done most conveniently by working directly with cut-diagrams, rather than amplitudes, because this facilitates the use of the same methods as for loop integrals. Thus, the method presented here is not restricted to a small number of special cases. It provides us with a consistent systematic computational scheme that can be applied to a wide variety of processes.

\section{Acknowledgements}

The work of M.B. and N.K. is supported in part by the DFG Sonderforschungsbereich/Transregio 9 "Computergestützte Theoretische Teilchenphysik"

\section{REFERENCES}

1. M. W. Grünewald et al., hep-ph/0005309.

2. M. Beneke, A. P. Chapovsky, A. Signer and G. Zanderighi, Nucl. Phys. B 686, 205 (2004) hep-ph/0401002; Phys. Rev. Lett. 93, $011602(2004)$ hep-ph/0312331.

3. A. P. Chapovsky, V. A. Khoze, A. Signer and W. J. Stirling, Nucl. Phys. B 621, 257 (2002) hep-ph/0108190.

4. M. Beneke and V. A. Smirnov, Nucl. Phys. B522 (1998) 321 hep-ph/9711391.

5. W. Beenakker, F. A. Berends and A. P. Chapovsky, Nucl. Phys. B 548, 3 (1999) hep-ph/9811481;

A. Denner, S. Dittmaier, M. Roth and D. Wackeroth, Phys. Lett. B 475, 127 (2000) hep-ph/9912261; Nucl. Phys. B 587, 67 (2000) hep-ph/0006307;

S. Jadach et al., Phys. Rev. D 61, 113010 (2000) hep-ph/9907436.

6. W. E. Caswell and G. P. Lepage, Phys. Lett. B 167, 437 (1986);

G. P. Lepage et al., Phys. Rev. D 46, 4052 (1992) hep-lat/9205007.

7. C. W. Bauer, S. Fleming and M. E. Luke, Phys. Rev. D 63, $014006 \quad$ (2001) hep-ph/0005275;

C. W. Bauer, S. Fleming, D. Pirjol, and I. W. Stewart, Phys. Rev. D63, 114020 (2001) hep-ph/0011336;

C. W. Bauer, D. Pirjol, and I. W. Stewart, Phys. Rev. D65, 054022 (2002) hep-ph/0109045;

M. Beneke, A. P. Chapovsky, M. Diehl and Th. Feldmann, Nucl. Phys. B 643, 431 (2002) hep-ph/0206152.

8. V. S. Fadin, V. A. Khoze and A. D. Martin, Phys. Lett. B 311, 311 (1993).

9. A. Pineda and J. Soto, Phys. Rev. D 59, 016005 (1999) hep-ph/9805424. 